\title{
Using PTES and open-source tools as a way to conduct external footprinting security assessments for intelligence gathering
}

\author{
Bruno Dinis ${ }^{1}$, Carlos Serrão ${ }^{2}$ \\ Department of Information Science and Technology, School of Technology and \\ Architecture \\ ISCTE-IUL/ISTAR-IUL \\ Av. das Forças Armadas, 1649-026, Lisbon, Portugal
}

\begin{abstract}
The first phase in a security assessment activity (legitimate or not) consists in the information gathering procedures that need to be conducted about a specific target. Information gathering, also known as footprinting, is the process of collecting all available and accessible information about a specific target to assess. While conducting a security assessment, this is one of the most important stages and usually involves the examination, collection and classification of large volumes of data from the target. The Penetration Testing Execution Standard (PTES), provides the description of the processes that are necessary to conduct penetration-testing assessments in a generic and integrated manner in all the different stages that compose such penetration testing process. However, the particular focus of this article consists in the analysis of the standard and its recommendations on what concerns footprinting processes and how to provide some contributions in terms of the practical applicability, namely on the usage of open-source footprinting applications, in the implementation of PTES recommendations.
\end{abstract}

\section{Introduction}

When a criminal plans a bank robbery, he doesn't simply walks through the bank front doors and yells: "This is a robbery! Give all your money!". In a vast majority of the situations this simply would not work, and would end up with the thief being arrested and put behind bars. Usually a bank robbery takes some planing and preparation, with the criminal studying its target and collecting as much information as possible, until it finds its weakest points to explore. This is as true for a bank as it is for digitally-based information assets.

This information gathering process is an important stage of a systems security assessment. It allows security professionals conducting testing to collect all the necessary information about a specific target or a set of targets that will allow them to have a full insight of the planned targets prior to any security assessment taking place. This is a crucial stage in a specific type of security assessment known as penetration testing. Penetration testing professionals (testers), also known as "ethical" or "white hat" hackers, are tasked with discovering information security vulnerabilities that might be potentially exploited by an attacker. Knowing our own vulnerabilities and weaknesses before our enemies has always been one of the best ways to create the appropriate strategies to cope with these weaknesses and actually be prepared to create protection measures against them [20].

The Penetration Testing Execution Standard (PTES) has recently emerged as one of the most known community-driven penetration testing framework. This framework, although still in early development stage, provides a structured methodology to a motivated community aimed to openly specify what penetration assessments are and which are the steps involved in penetration testing processes. One of the most relevant objectives of PTES is to provide potential customers with a benchmark that can be used to determine the quality of tests carried out for them by contracted penetration testers. Throughout a conventional expression and scope to accomplish penetration tests, this framework aims to increase the global quality of penetration testing assessments and specially supporting businesses to define what they need to perform a security assessment to their systems and what to expect from the different penetration testing phases in terms of results.

This methodology [1] clearly identifies seven specific, different but complementary stages. It allows a normalised approach to the following defined set of stages (Figure 1):

- pre-engagement interactions

- intelligence gathering

- threat modelling

- vulnerability analysis

- exploitation

- post-exploitation

- and reporting [1]. 


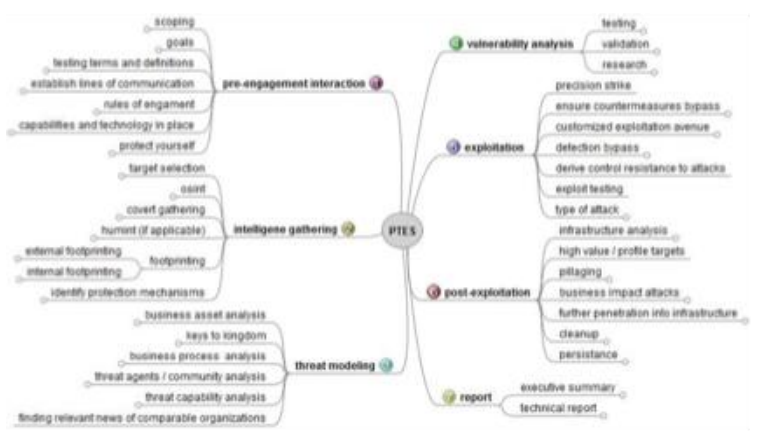

Figure 1. An overview of the PTES recommendation

Throughout this article, we will focus primarily on the second stage of the PTES recommendation intelligence gathering - while providing some specific examples of open-source tools that can be used to implement information gathering techniques. We also provide some examples on how the PTES recommendation can be fulfilled to conduct external footprinting, that is conducted from the outside of the evaluating targets.

One of the weakest points of PTES, although extremely exhaustive on the process description, fails to provide a mapping between these processes and its practical applicability on the field. One of the objectives of this article is to provide a contribution to PTES while offering a mapping for the intelligence gathering process inside PTES and some of the open-source tools that can be applied to accomplish this stage.

In this article we start by providing an overview of the different stages that compose the PTES, while offering a small description of each of the different stages. The second part of the article describes in more detail the PTES footprinting processes, while it provides more details about tools and methods that might be used to conduct this stage. Finally, at the end of the article we present some conclusions from our work.

\section{PTES Stages overview}

The PTES establishes several stages where security audits need to be conduit and provides a set of guidelines that need to be followed to meet the objectives of each of the stages (or the expectations of potential clients) [1].

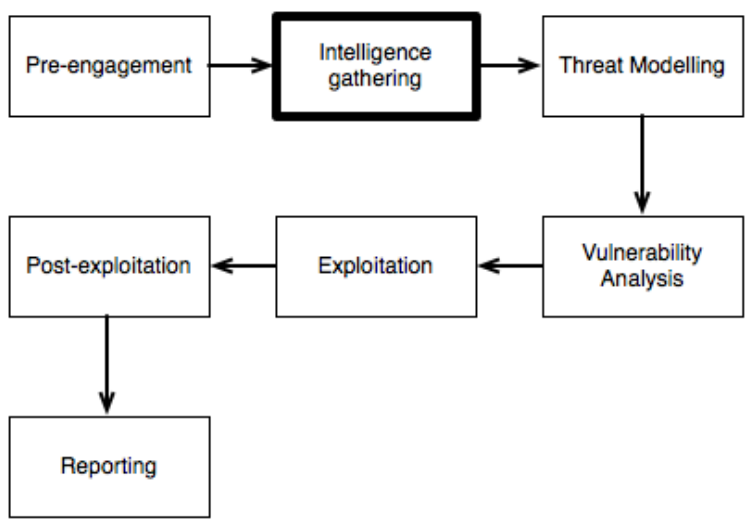

Figure 2. The different stages of the PTES

These stages and guidelines, presented in the image (Figure 2), can be resumed in the following:

1. Pre-engagement. The first phase describes all the pre-engagement actions and scope descriptions. Scoping is possibly one of the most significant and frequently ignored sections of a penetration test. It provides an important guideline about what to consider or not on the test.

2. Intelligence gathering. In this stage, is specified the information collection necessary to produce an intelligible representation of the business and its own procedures. All the data picked during this stage will contribute to guide all the evaluation of the possible organisation vulnerabilities. It will also determine what kind of IT infrastructure is used, information about workers, products and operations.

3. Threat modelling. Based on the previous stages, this phase covers the fundamentals of the threat modelling. In a conventional description this segment outlines a risk modelling approach as mandatory for a precise implementation of the tests. This framework does not endorse a specific model, however compels that the standard used must be reliable in terms of its depiction of risks, their potentials, their limitations for the organisation being analysed, and the skill to constantly be requested to future penetration tests with the similar outcomes.

4. Vulnerability analysis. Also known as vulnerability assessment, this level describes the key subjects the vulnerability analysis must cover. It is a method that describes and categorises the security vulnerabilities, while defining and sorting network or system resources that can provide different levels of importance to these resources. In addition, recognising conceivable threats to each resource develops a plan to deal with the most severe possible problems. The major objective at this point is to compile a high value target list and attack vectors in order to determine the impact on the organisation in successful attacks. Conceiving this target list will also define and fulfil security methods to diminish the costs if an attack occurs.

5. Exploitation. This represents the phase, where the attacker effectively exploits the target. However, it is mandatory to take attention on all the preceding tasks in order for this one to be fruitful. Exploitation 
is the triumphant manipulation or misuse of defenceless equipment, service or somebody to eventually gain access to data or information otherwise unreachable. This process involves the penetration tester to have persistently investigated and proved all potential threats to the target by effectively leveraging all earlier obtained knowledge of the target. This phase concentrates exclusively on establishing access to a service or machine by bypassing safety boundaries. If in the previous stage, vulnerability analysis were achieved accurately, this stage would be organised and accurate. The foremost effort is to detect the leading entrance point into the organisation system and to recognise high value target assets.

6. Post exploitation. After the penetration tester has control over an objective target and approaches the post-exploitation stage, and the main goal is to stay undetected and got future control on the exploited systems. Starting the exploiting methods all over again is not a good practice. A standard procedure is covering used scripts to regain access when needed. Thus this technique prepares the exploited system to take advantage of it in the next time. Other method of post exploitation is attacking the system from the exploited machine, covering all the eventual tracks of the manipulative activities. The reason of this last phase is to verify if the target compromised is worthless or not and provide access of the system for further use. The significance of the compromised system is established by the sensitivity of the data stored on it and the valuable machines in further attacks to the network [1].

7. Reporting. To summarise his work the penetration tester should create a document where he uncovers all security vulnerabilities discovered during the system audition. This document is projected to outline the base principles for penetration testing and report all the vulnerabilities discovered to those who are responsible for that. Network and system administrators must be warned about infrastructure vulnerabilities, developers must be advised about weaknesses in their code design [2]. Don Williams refers that "writing a penetration testing report is an art that needs to be learned to make sure that the report has delivered the right message to the right people" [3]. This record concludes the penetration testing in the organisation and should be taken seriously by the management and administration to mitigate future weaknesses and exposures in their systems.

\section{PTES versus other methodologies}

PTES is just one of the alternative methodologies that can be used. Two other open and free methodologies can also be applicable to the same type of activities:

- Open Source Security Testing Methodology Manual (OSSTMM)
- Information Systems Security Assessment Framework (ISSAF).

All of these methodologies help security professionals to define the best strategy that will guide auditors on the objectives definition and fixation, define the customer exigences and choose the most adequate types of tests. They supply the general security guidelines and procedures for all the organisation assets.

\subsection{Open Source Security Testing Methodology (OSSTMM)}

The OSSTMM methodology provides a complete set of security tests that are free from political and organisational influences. It emphasises particularly the technical details of most of the systems that need to be tested, what to do before, during and after a security analysis, and even how to calculate the results of the evaluation. Being an open methodology, it allows the free flow of information and intellectual property, including clarifications to project planning, quantifying the results, and standards for those who will perform the tests. The main objective of the OSSTMM is to provide an acceptable methodology for an accurate security characterisation through a substantial and reliable analysis [21]. This way, it fits admirably to most audits, penetration testing, security and vulnerability assessments. Its target studies are divided into five parts: information and data testing, supervision of staff, security awareness levels, fraud and social engineering [22]. Are also documented test cases in computer networks, mobile devices and wireless networks, physical and logical security access controls. Although there are progresses made for the release of version 4, this methodology is still currently at version 3 , becomes more mature as more users around the world contribute to its integrated development in the ISECOM working group [23].

\subsection{Information Systems Security Assessment Framework (ISSAF)}

The ISSAF is another open methodology for security testing in applications and networks provided by the non-profit organisation OISSG (Open Information Systems Security Group). Its disposition was classified in various fields as a way to conduct a safety assessment. The ISSAF was developed with the objective to focus on two areas of security testing, technical and management. On the one hand, the technical area defines a set of rules and procedures to be followed, creating an adequate security assessment process, on the other hand aligns the management area with the best practices to be followed throughout the analysis process [24]. Its methodology is defined into four different areas: safety nets, hosts, applications and databases. Each of this areas has general instructions that are 
effective and flexible to any organisational structure, simplifying the implementation of this methodology. ISSAF contains a rich set of technical evaluations to test the number of different technologies and processes. Being a more flexible methodology and updated information, best practices and administrative care to complement the security assessment program, it becomes quite versatile and powerful. The auditor can align its methodology with any other similar, thus combining the advantages of each. This framework, although it is still in a stage of maturity growing, confers a high value proposition, which guarantees the security of the organisational infrastructure, evaluating existing security controls and their critical weaknesses. It also has operations management controls, physical security assessment, penetration testing, incident management and other.

\section{Intelligence gathering}

In this phase we proceed to the collection of information in order to obtain a logical representation of the organisation. It must be detailed and specific contributing to a more in-depth assessment of vulnerabilities. The more vulnerabilities are found, more attack vectors exist during the penetration test and most likely has the system to be explored. Before starting the technical safety assessment, it is important to examine and understand the target environment.

\subsection{Target selection}

Depending on the penetration testing to be conducted, the target may or may not be revealed. If the test is "Whitebox" all targets are known while in the "Blackbox" testing there is no advance knowledge of the technological infrastructure.

\subsection{OSINT - Open Source Intelligence}

The Internet is a great source of knowledge. The information can be accessed anywhere in the world through search engines that locate all the information researched. With the ease in data finding, one can draw not only personal but also organisational profiles. With the application of certain search techniques it may be possible to obtain data about the organisations and get job listings, financial reporting and information about the organisational structure. This valuable information is useful for social engineering attacks, if it is one of the objectives set initially.

\subsection{Covert gathering}

Despite the covert information gathering process is not well defined in PTES, it is common to define this concept as a set of unconventional activities to get extra information on a particular target. Although not illegal, these processes often include research into bins (dumpster diving), as a method of gathering information. The identification of physical security mechanisms, wireless networks research and radio frequency signals can also be identified as Covert Gathering techniques.

\subsection{Human Intelligence (HUMINT)}

This is a concept that is related to social engineering. It is a phase where there is direct interaction with the target or people related to it. The methodology for obtaining human intelligence always involves physical or verbal interaction. For example, by responding to an ad with a false identity and the competencies requested by the target organisation, the auditor will interact directly with members of the HR team or even with the coaching staff. You might as well collect information through the direct observation of the physical security controls (doors, security guards) or digital (fingerprint readers, access controls) as well as internal or behaviour patterns such as the "dress code" used in the organisation.

\subsection{Footprinting}

At this stage there is no direct interaction with the target. In this stage it is expected to know the IP address and number of connected machines, which are the open ports and services in order to realise which is the best attack vector that can be used for penetration testing systems. Gathering information about the version of the systems and services will be used in vulnerability identification during the research phase. There should be an efficient research work in order to have the greatest certainty of targets to carry out the attacks. This footprintg phase will be further detailed in this paper.

\subsection{Protection mechanisms identification}

The auditing team predicts the use of protective mechanisms of the logical perimeter, such as firewalls, IDS / IPS. These controls can be used to protect and preserve the other technological infrastructure. It should also be noted at this stage that may be triggered alarms, it is necessary to use prudence in the use of information-gathering tools.

\section{Footprinting in PTES}

Reconnaissance and footprinting are similar concepts. Nevertheless it is essential to understand the differences between them. Reconnaissance can be defined as the initial phase of the security assessment activity where attackers will attempt to learn and collect as much as possible evidences about the target. This information is gathered to characterise the infrastructure under investigation. 


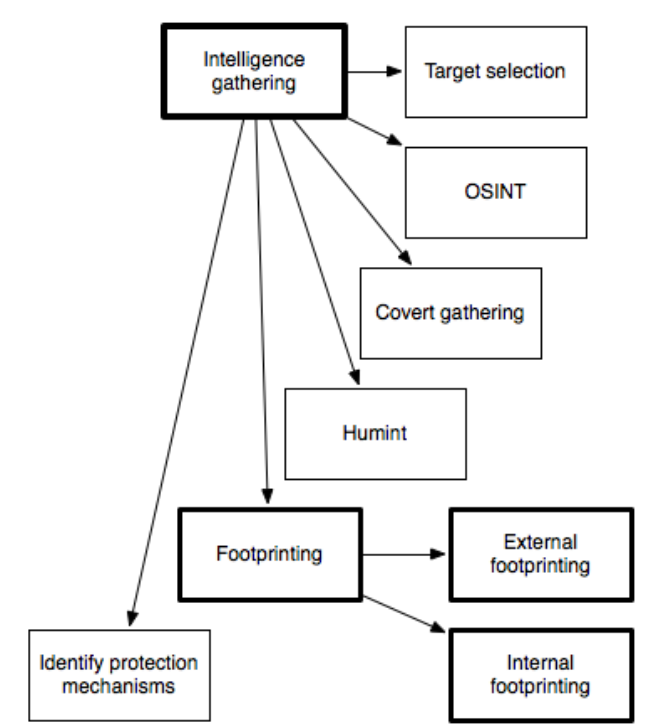

Figure 3. The specific stages of the intelligence gathering stage of PTES where foot printing plays and important role - both external and internal

Although footprinting can be labeled as a passive and non-intrusive reconnaissance technique, tolerating the potential attackers to accumulate all potential information about the target without the requisite of employing forceful reconnaissance methods [4], can be quite dangerous and imprudent (this is usually the first step for an attack). However, according to the PTES framework, footprinting can be both passive and active (Figure 3). For instance, conducting a review of the website of an organisation is a type of passive footprinting, while intensive scanning of machines and networks can be considered as an example of active footprinting. They both can be evasive and silent techniques and it is important to know when to apply them. The PTES framework defines the footprinting technique as being part of the second overall phase of the penetration testing procedure - the informationgathering phase. Divided into two different spheres, the footprinting could be performed externally or internally. In the external mode, the framework identifies the customer external ranges, conducts passive reconnaissance, active footprinting and also establishes an external target list. On the other hand there is also the internal footprinting that covers three main topics: the passive reconnaissance, customer internal ranges identification and the active footprinting.

As it was previously referred the focus of this paper is on external footprinting that follows the best practices described by the PTES, and on the mapping of open-source tools and techniques that allow the implementation of such good practices. In the following sections of this paper, some examples of the execution of external footprinting are provided [13].

These examples were performed in a controlled and authorised network environment using a virtualised laboratory. In accordance to the law, in most countries, some of the tests and techniques when used in a non-authorised manner in nonauthorised targets are considered a serious crime.

\subsection{External footprinting}

During an external penetration test, it is necessary to perform an assessment on all Internet accessible assets (all the organisation assets that are accessible from the exterior of the organisation using an Internet connection). This way, an evaluation of the target security from an outsider perspective is conducted. The external footprinting process of intelligence gathering stage comprises collecting answers from the objective target created from an exterior point of view. The purpose is to accumulate as much information about the target as possible. The PTES framework covers the techniques that are presented in the following sections.

\section{Identifying customer external ranges}

The intelligence gathering stage is critical for the penetration testing execution. The main objective during the security audit is fixing all the possible target hosts. With these hosts in mind the audit can be launched.

\section{Passive reconnaissance}

From the external point of view, the passive reconnaissance is based on the search of publicly available information about the target on the Internet. This is sometimes also known as OSINT (opensource intelligence) [14]. This process of information collection about an intended target occurs silently without any knowledge of the victim. Any valuable search engine (such as Google, for instance) can support security auditor's work offering lots of background information about almost any target [5][15]. Since the web address (or IP address) is public, loads of sensitive information can be found while performing this kind of passive exploration.

Conducting a search in the chronologic information hosted in the website of the target can also prove to be quite useful. The public online service "Internet Archive" [6] saves website information since 1996 and it makes possible to seek information about the target since that date. For instance, it is possible to search in this archive for a specific website URL and it will reveal how many times the website has been crawled since it was launched by the first time [6].

Usually, passive reconnaissance can also embrace physical inspection of an organizational structure, seeking over wasted machines or other equipment in an effort to locate hardware that may include valuable information. Other techniques may also include dumpster diving or simply eavesdropping the network. 


\section{Active footprinting}

Such as the passive counterpart, active footprinting consists in collecting information about an intended target system. Also known as active reconnaissance, this concept usually includes port scanning in order to discover flaws in the target system, like vulnerable ports or services that could be used to compromise the firewall or routers and leverage the exploits. Nmap is an open-source network mapper tool [16] that can be used for network discovery and security auditing. Nmap is capable of scanning network ranges. In the following example, Nmap can be used to find which are the active assets on a network, which are the ports that are open or closed on these assets also, which are the services that are running on such ports. Nmap can be used with different combinations to extract even more information from the targets.

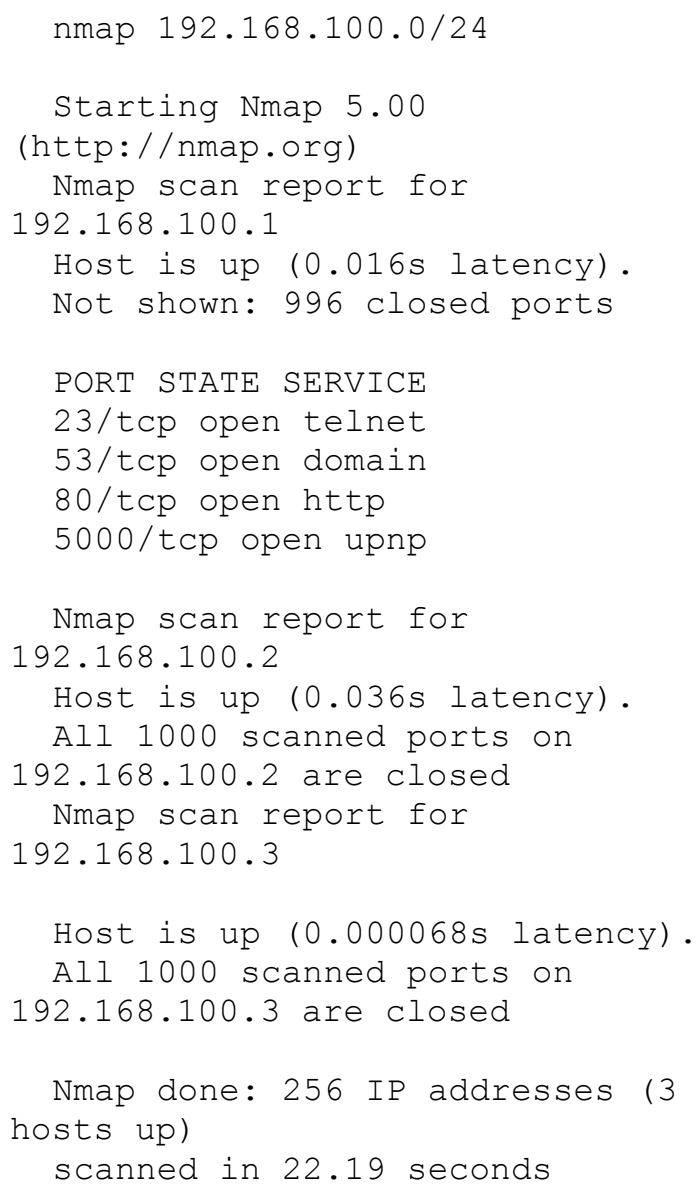

The method of exploiting the target can then be carried out once the auditor has established a way to access the system from an outside perspective. Banner grabbing is another well-known technique for conducting external footprinting of a target system. This technique allows the security auditor to find out which service or application is up and running and on which port. This is the process in the audit that simulates an attacker attempts to locate an application version installed in victim's machine and find any known exploits or vulnerabilities for that specific combination (application and version). With this information, an attacker could exploit known vulnerabilities using specific exploits [7]. There are online public databases of vulnerabilities, indexed by service/software and version that allow access to millions of identifiable vulnerabilities (for instance the Common Vulnerabilities and Exposures (CVE) database) [17].

The example that is provided next allows the security auditor to learn which service the SNMP server is using. Using a simple telnet session in all the reported open ports and waiting for the response banner will provide valuable information. For example, this is the response banner of a SMTP server running PRTG. If the services and versions are revealed during the penetration test and if there are known vulnerabilities for these services/versions then they could be easily exploited by an attacker.

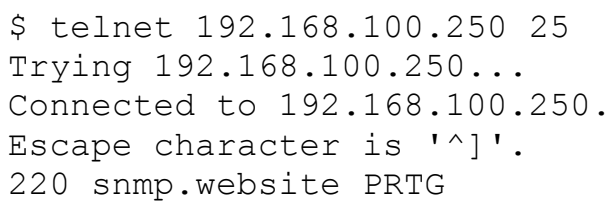

Another technique that is mentioned by PTES framework is SNMP Sweeps [18]. Using this allows the discovery of huge volumes of information about a specific system or actually it also allows the compromise of a remote device. When interrogating through SNMP service, there is a MIB (Management Information Base) that permits to request information to the target machine. Metasploit [12] is a free penetration testing software and offers a catalogue of default MIBs database. Applying them, would probe the target machine for extra info. Its auxiliary module could be applied to the following example, showing what IP addresses are using default community strings, which can be easily exploited.

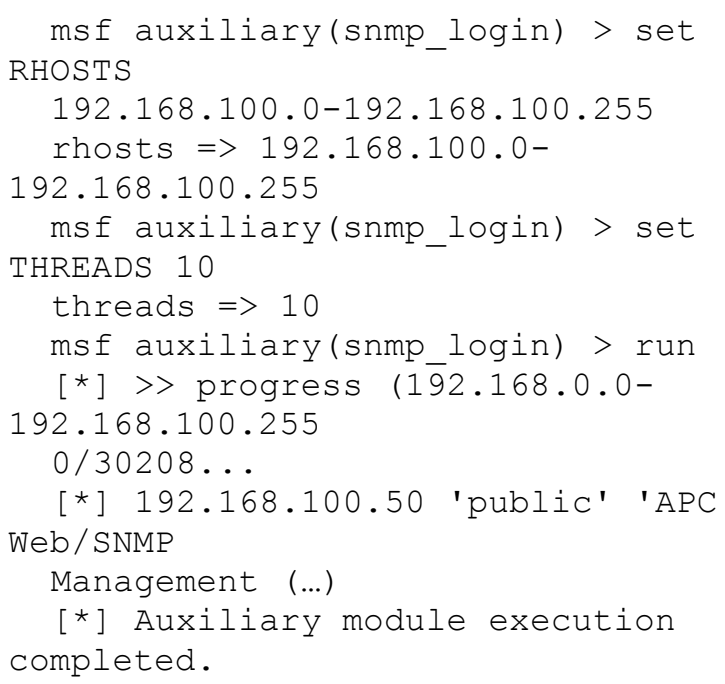


Zone Transfers are a vital source of data about the network. This technique is used to allow backup DNS servers to synchronize with their primary servers by querying simple requests for the DNS records for a specified domain. For each of the requests it is provided all the known information about a single domain, although it can be used to gather information about the servers (huge leakage of information). Zonetransfer.me is a domain registered to show how DNS zone transfer works, explaining the security problems concerned [8] and how an attacker can harvest information about an organization DNS.

dig axfr ans12.zoneedit.com

zonetransfer.me

$<<>>$ DiG 9.7.3-P3 <<>> axfr

ans12.zoneedit.com zonetransfer.me

(1 server found)

global options: +cmd

zonetransfer.me. 7200 IN NS

ns16. zoneedit.com.

zonetransfer.me. 7200 IN NS

ns12.zoneedit.com

zonetransfer.me. 7200 IN A

217.147 .180 .162

The Non-Delivery Report/Receipt (NDR) or the SMTP bounce back is a notification about a mail system. It is used typically to get extra information about the email system, getting the headers and sometimes the infrastructure details by composing a faulty email to the target domain and getting the report on this delivery failure. This is an issue that can simply be simulated by creating a bogus email address within the target's domain. Using this method it would be possible to uncover the email server IP.

Here's an example of a common email provider header:

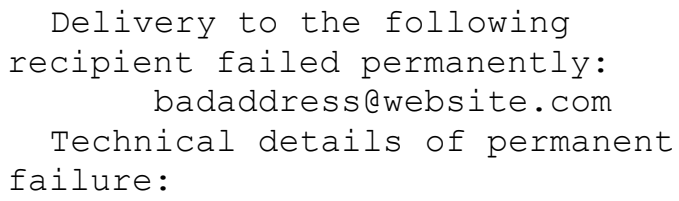

We tried to deliver your message, but it was rejected by the server for the recipient domain "Real IP Email Server Address Displayed"

The error that the other server returned was: 550 badaddress@website.com: Recipient address rejected: User unknown in local recipient table.

Another method mentioned in the PTES is DNS Discovery. Normally mentioned as "whois" technique and widely used on Internet, this routine allows queries to remote databases for domain registration information. After collecting all the information needed using the above methods, the auditor could query the DNS using some opensource tools.

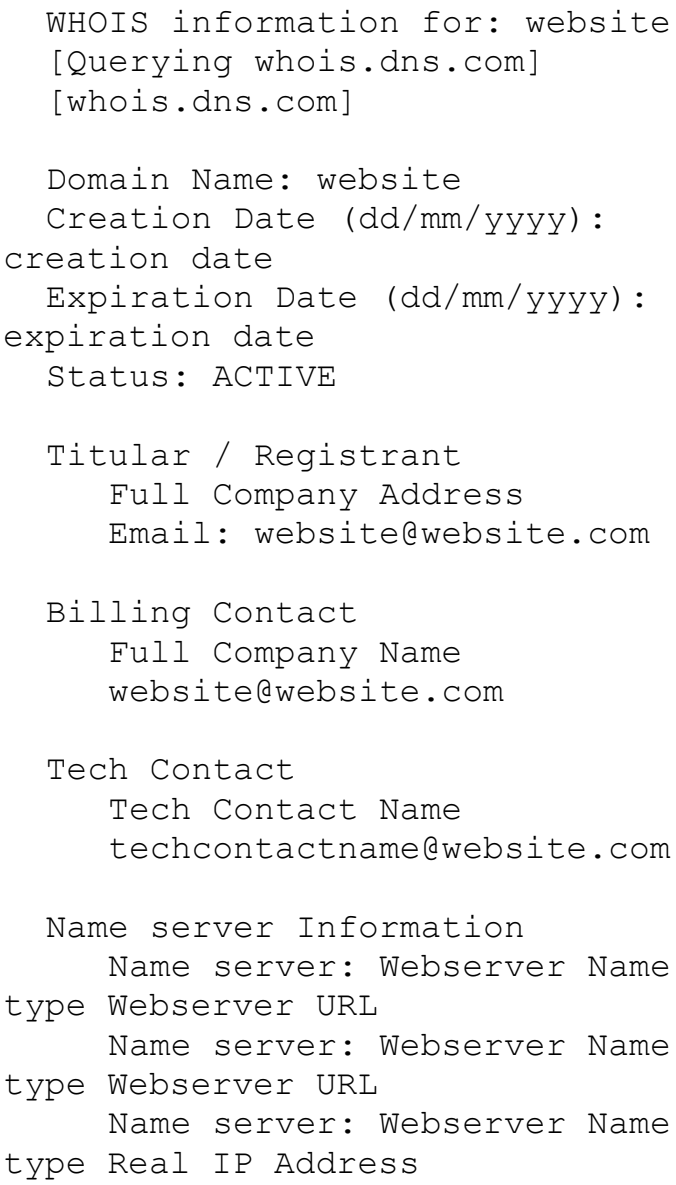

If the Domain Name Server points the address to his associated IP address, the Reverse DNS method can be handled to achieve valid server names by trying the server with several IP addresses to check if it returns any outcomes. If it does resolve the name then the results are returned. There are some available examples on how to how to configure the Reverse DNS [9].

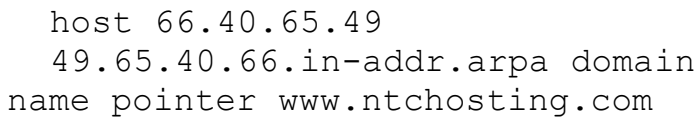

After discovering the initial information, a DNS brute force technique [19] can also be used in the information gathering stage according to the PTES framework. Forcing the queries to the DNS server, this tool seeks for misconfigurations about the DNS server to allow users to perform Zone Transfers as it was mentioned before.

Web Application discovery is also a procedure meant to uncover installed web applications on the target system, and vulnerabilities that could be exploited by identifying those applications. To verify which is the OS running on the target system (OS fingerprinting), the auditors can benefit from several open-source tools (or even some web-sites) that are 
available on the Internet. For instance, the Netcraft website as a service called "What is that site running" that identifies the operating system and the server software that a given web site is running. To use this tool, it is simply necessary to enter the target URL name on Netcraft webpage and all the details are revealed. Besides operating system detection this company offers more vulnerability analysis tools [10].

$$
\begin{aligned}
& \text { Search for "website" } \\
& \text { OS Server Last Change IP } \\
& \text { Linux Apacher 22-Jun-2013 } \\
& \text { A.B.C.D }
\end{aligned}
$$

The final technique that is enumerated on the PTES framework is the Virtual Host Detection \& Enumeration. This technique is used to discover the host names related to a given IP address. HostMap is an open-source discovery software designed to detect hostnames and virtual hosts in the network and performs vulnerability evaluations and penetration testing. This tool assists the auditor on the exploration of several techniques to specify and enumerate all the hostnames related with a specific IP address [11].

Establishing external list

The final result of the information-gathering phase is creating external inventory with all the information collected and of all the uncovered vulnerabilities. This is one of the areas where the PTES framework still lacks on information. The mapping of all the versions of the applications/services/assets gathered, consequence of the exploitation of some of its vulnerabilities, it is critical. This is the information that probably an attacker would use to compromise some of these assets. With this information, an attacker could label all the existing patch levels for all those found applications, interrogating the system with random vulnerability scanners.

An attacker can also look for weaker applications installed, find a breach in its security and then implement the way to exploit it. Once inside the infrastructure he can leverage the privileges and seek for storage setup evidences, virtualisation platforms and virtual machines running on the systems infrastructure. The attacker can identify the lockout threshold to perform the attack identifying weaker ports and outdated systems. Finding the lockout threshold of a validation service will permit the attacker to guarantee that some hacks do not deliberately lock out valid users during the testing [1].

\section{Conclusions}

The evolving Penetration Test Execution Standard (PTES) is a collaborative effort, supported by a wiki-based system, meant to offer a penetration testing security service a set of well-defined and standardised practical guidelines. Although still in an early stage, the footprinting phase holds a critical significance in the information-gathering phase. In the article all the different seven phases were briefly described, although the article focus was in the footprinting process. Footprinting reveals the work of information gathering that needs to be carried out before any penetration testing is actually conducted. In this article, some methods based on open-source tools that are capable of gathering information about a target testing system, were also presented. These methods can reveal potential vulnerabilities and attack points on the target's infrastructure. The usage of these open tools will ensure the applicability to the PTES framework, complementing its information and covering one of its weakest aspects.

As it was demonstrated in this paper, the information gathering stage is of extreme importance because it ensures that the next stages on the penetration testing are actually executed in a proper manner, targeting the most relevant vulnerabilities and addressing the proper targets - therefore minimising the time and effort of the testing itself.

The penetration test methods that were described in this paper are extremely important to secure and mitigate the risk of compromising the security so they are essential to conduct proper penetration testing in a way to conduct exhaustive security audits. An organisation should be conscientious that it is important to know its own weaknesses and vulnerabilities before an attacker, in order to be able to raise the necessary fences to keep intruders out. Otherwise, it has little change to resist an attack [20].

\section{References}

[1] The Penetration Testing Execution Standard. (2012). Retrieved July 02, 2014, from http://www.penteststandard.org/index.php/Main_Page

[2] Wren, C., Reilly, D., \& Berry, T. (2010). Footprinting: A Methodology for Auditing eSystem Vulnerabilities. In 2010 Developments in E-systems Engineering (pp. 263267). IEEE. doi:10.1109/DeSE.2010.49

[3] Williams, D. (2005). A Guide to Discovering Web Application Insecurities, Before Attackers Do. SANS Institute. Retrieved July 02, 2014, from http://www.sans.org/reading-

room/whitepapers/webservers/guide-discovering-webapplication-insecurities-attackers- 1557

[4] E-CQurity (2011). Footprinting Encored. Retrieved July 02 , 2014, from http://www.e-cq.co.th/wp/footprintingencored.pdf

[5] Mansfield-Devine, S. (2009). Google hacking 101. Network Security, 2009(3), 4-6. doi:10.1016/S13534858(09)70025-X

[6] Internet Archive: Digital Library of Free Books, Movies, Music \& Wayback Machine.. Retrieved July 02, 2014, from https://archive.org/

[7] Lanz, J. (2003). Practical aspects of vulnerability assessment and penetration testing. Rma Journal, 85(5), 52-57. 
[8] ZoneTransfer.me - DigiNinja. (2013). Retrieved July 02, 2014, from

http://digi.ninja/projects/zonetransferme.php

[9] How does the Reverse DNS work? (2013). Retrieved July $\quad 02, \quad 2014, \quad$ from http://www.ntchosting.com/dns/reverse-dns.html

[10] Netcraft | Internet Research, Anti-Phishing and PCI Security Services. (2013). Retrieved July 02, 2014, from http://www.netcraft.com/

[11] hostmap - the automatic hostnames and virtual hosts discovery tool. (2013). Retrieved July 02, 2014, from http://hostmap.lonerunners.net/

[12] Jaswal, N. (2014). Mastering Metasploit (p. 378). Packt Publishing Ltd.

[13] Faircloth, J. (2011). Penetration Tester's Open Source Toolkit (p. 441). Elsevier.

[14] Glassman, M., \& Kang, M. J. (2012). Intelligence in the internet age: The emergence and evolution of Open Source Intelligence (OSINT). Computers in Human Behavior, 28(2), 673-682. doi:10.1016/j.chb.2011.11.014

[15] Billig, J., Danilchenko, Y., \& Frank, C. E. (2008). Evaluation of Google Hacking. In Proceedings of the 5th Annual Conference on Information Security Curriculum Development (pp. 27-32). New York, NY, USA: ACM. doi:10.1145/1456625.1456634

[16] Nmap - Free Security Scanner For Network Exploration \& Security Audits. (n.d.). Retrieved July 02, 2014, from http://nmap.org/

[17] CVE - CVE List Main Page. (n.d.). Retrieved July 02, 2014, from https://cve.mitre.org/cve/

[18] Herrero, Á., Corchado, E., \& Sáiz, J. M. (2005). Identification of anomalous SNMP situations using a cooperative connectionist exploratory projection pursuit model. In Intelligent Data Engineering and Automated Learning-IDEAL 2005 (pp. 187-194). Springer Berlin Heidelberg.

[19] Sutton, M., Greene, A., \& Amini, P. (2007). Fuzzing: brute force vulnerability discovery. Pearson Education.

[20] Tzu, S. (2013). The art of war. Orange Publishing.

[21] Herzog, P. (2014), Open Source Security Testing Methodology Manual (OSSTMM), Retrieved December 2014, from http://www.isecom.org/research/osstmm.html [22] Pavkovic, N., Perkov, L., (2011). Social Engineering Toolkit - A systematic approach to social engineering. 34th International Convention MIPRO, Volume 1, 1485 1489.

[23] Prandini, M., Ramili, M. (2010). Towards a practical and effective security testing methodology. In Computers and Communications (ISCC), 2010 IEEE Symposium. Riccione, Italy, 22-25 June 2010. Univ. di Bologna, Italy: IEEE. 320 - 325.

[24] Jackson, C. (2010). Network Security Auditing. 1st ed. Indianapolis, USA: Cisco Press. 